\title{
Advances in Clinical Neuroimaging
}

\author{
Joseph I. Tracy ${ }^{1}, \mathrm{PhD}, \mathrm{ABPP} / \mathrm{CN}$; Gaelle Doucet ${ }^{2}$, PhD; Xaiosong $\mathrm{He}^{2}$, PhD \\ Dorian Pustina², PhD; Karol Osipowicz ${ }^{2}, \mathrm{PhD}$ \\ ${ }^{1}$ Department of Radiology, Thomas Jefferson University, Philadelphia PA \\ 1,2Department of Neurology, Thomas Jefferson University, Philadelphia PA
}

\begin{abstract}
A paradigm shift has occurred in Neuroscience, with most brain diseases, even those considered focal, now viewed as having repercussions throughout the whole brain. As one might suspect these brain networks effects are quite complex, so multiple neuroimaging techniques are needed to view them.
\end{abstract}

Structural magnetic resonance imaging (MRI) is used to determine the health of brain tissue, particularly in the cortex. Resting-state functional connectivity (rs-fMRI) is used to identify cortical regions communicating with each other. Diffusion imaging can verify that any two regions are, indeed, anatomically connected through white mater fibers. Lastly, functional MRI can be used to define the specific cognitive information and processes the regions and networks may be communicating about. Data from all four imaging technologies can be gathered during one MRI scanning session with a minimal increase in scanning time and no increase in patient discomfort.

A key goal of our laboratory has been to create individual brain maps of cognitive functions that may be negatively affected by invasive brain treatments such as thermal ablation, brain stimulation, or resective brain surgery. Neurologic disease often leads to alterations in how and where the brain stores cognitive skills and behaviors. In some cases, changes in how the brain stores information can be so complete that the regional network implementing a function (e.g., expressive language) can be entirely discordant with established theories about the brain localization of function (e.g., Broca's, Wernicke's in the right not the left hemisphere; see Figure 1b). An accurate map of where in the brain key cognitive functions are located can help avoid removal of intact, functional tissue, and guide access routes so the surgeon can reach and remove the pathology. Such brain maps also save the neurosurgeon time because they can more rapidly identify the location of functional areas near the diseased or pathologic tissue that needs to be removed.

The important functions frequently mapped out include speech, receptive language, primary motor and sensory regions (Figures 2 and 3), and memory functions (Figure 4). Techniques, however, are being developed to map out emotion processing, problem solving, abstract thinking, planning/organization and behavioral control. Since the pathways connecting brain regions are as important to successful cognition as the regions themselves, diffusion tensor imaging provides a crucial roadmap of pathways to avoid during brain surgery, if at all possible. For instance, Figure 5 shows a diffusion image constructed to help avoid visual pathways in an epilepsy patient undergoing surgery for an epileptic region near the brain centers for vision. Likewise, Figure 6 shows the white matter pathways that help perform voluntary movements in a patient with a frontal tumor near the brain areas for movement. Lastly, Figure 7 shows the white matter tract (green) in an epilepsy patient connecting the two key brain areas involved in language.

Our laboratory has led advances in brain mapping, particularly in the area of resting state functional connectivity as applied to epilepsy (Tracy and Doucet, 2015; Tracy, 2015). Techniques such as rs-fMRI can be particularly useful in mapping out function in patients who may not be able to understand or cooperate with the instructions or demands of a standard fMRI task. Figure 8 displays the results of the first study to utilize functional connectivity to develop a method for identifying which hemisphere is most important for language, a method that does not require the patient to actually complete a language task (Doucet at al., 2014). We also produced the first resting state study to show that connections from the ictal (seizure) to the non-ictal (healthy) hemisphere work to control and confine seizures to a more limited region in the brain through inhibitory mechanisms generated by the healthy hemisphere (see Figure 9; Tracy et al., 2014).

An important clinical goal is the development of imaging tests that might tell us whether an individual is at risk for disease, or if a patient about to undergo surgery is likely to experience a good outcome. This predictive ability will require, however, an understanding of the principles that govern adaptive responses in the brain and lead to the restoring of lost cognitive functions. With this in mind, we developed a conceptual framework for integrating multiple imaging modalities toward the goal of identifying the brain regions involved in change (neuroplasticity) and network reorganization following brain injury (Figure 10; Tracy \& Osipowicz, 2011). The combined power of several imaging modalities can be used to predict outcomes prior to undergoing procedures such as brain surgery for epilepsy. For instance, Figure 11 shows how the combination of multiple imaging modalities was able to distinguish poor versus good outcome patients (i.e., those achieving seizure freedom), with diffusion tensor imaging serving as the best predictor (see Figure 11; Osipowicz et al., 2015). Currently, our lab is developing quantitative imaging algorithms for predicting such important clinical outcomes. As a start, we completed a recent study demonstrating the utility of a quantitative algorithm for determining from which side of the brain (hemisphere) an epilepsy patient's seizures might be coming (Pustina et al., 2015).

This work has the potential to alter clinical practice by providing justification for including multiple imaging methods into decision-making algorithms for brain surgery. The hope is to eventually develop imaging tests (biomarkers) that will be available on a presurgical basis to help predict the probability of surgical success, which will help both patients and their treating doctors decide on the appropriateness and risk of brain surgery. 
Clinical Neuroimaging
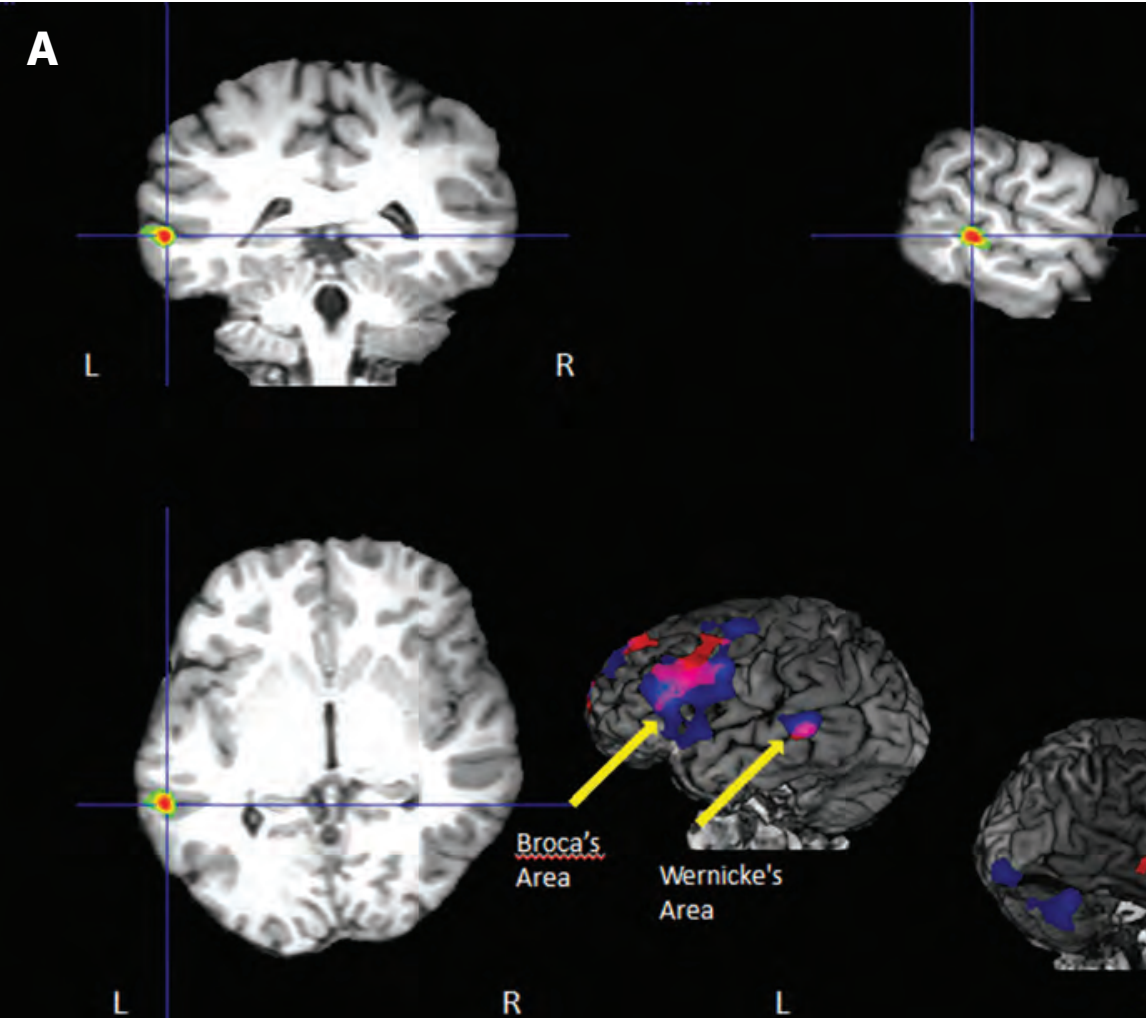

\section{Figure 1}

A. FMRI activation sites for a verb genera-

tion and sentence

completion task in an epilepsy patient with

\section{intractable temporal}

lobe epilepsy. The

pattern of activation in

the left hemisphere (see arrows pointing to key language sites, Broca's and Wernicke's areas) reflects left hemisphere language dominance. B. Activation for the same two tasks, in a different temporal lobe epilepsy patient. This pattern reveals atypical regions of activation, reflecting right hemisphere dominance for language.

L

R

L



$\mathrm{R}$

B
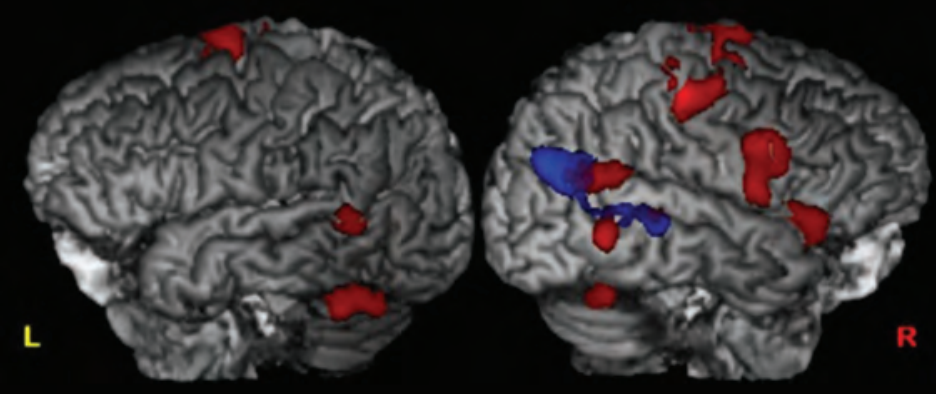

Verb Generation

Sentence Completion
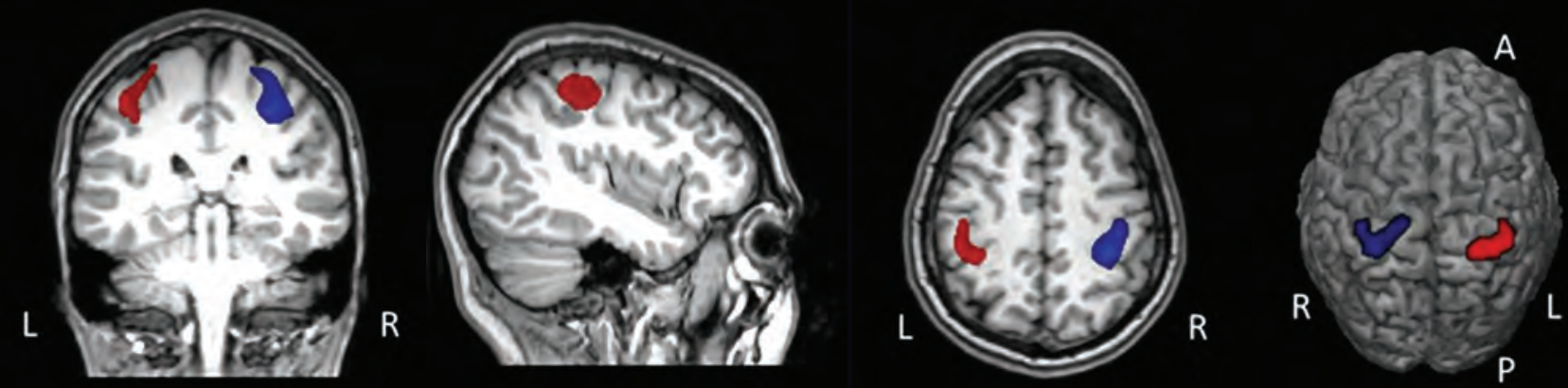

Left Hand Motor Right Hand Motor

\section{Figure 2}

FMRI activation in the normal brain locations associated with left and right hand motor movements. 


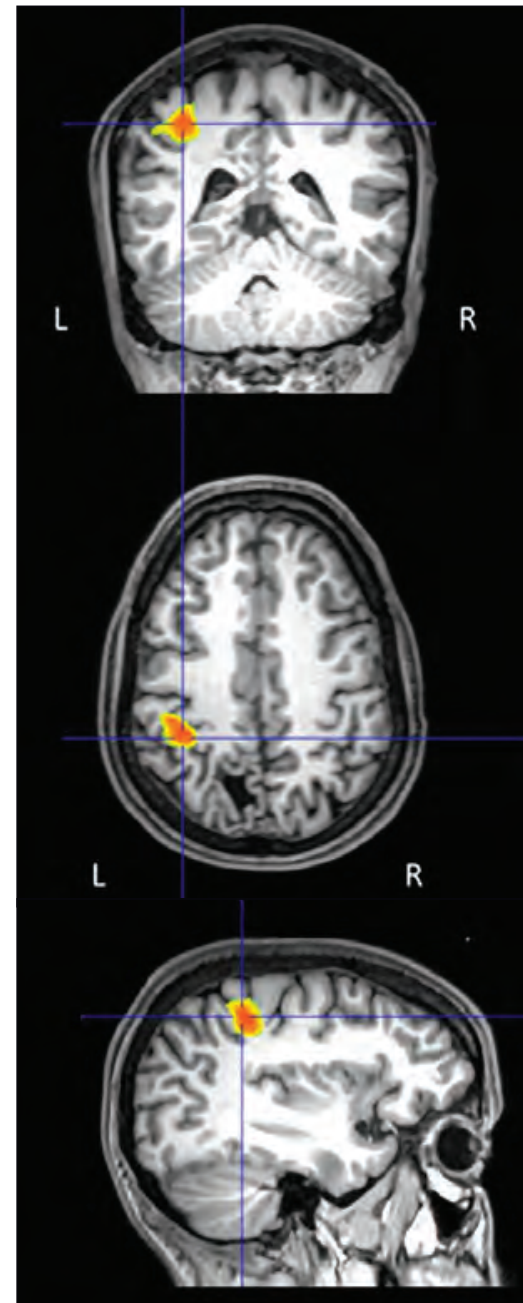

Right Hand Sensory

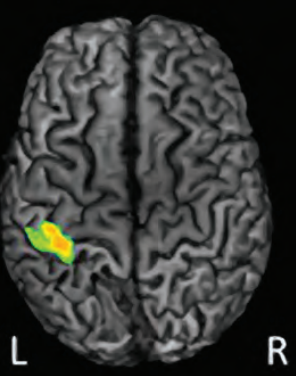

Figure 3

FMRI activation in a tumor patient revealing the brain area typically involved in right hand tactile stimulation.
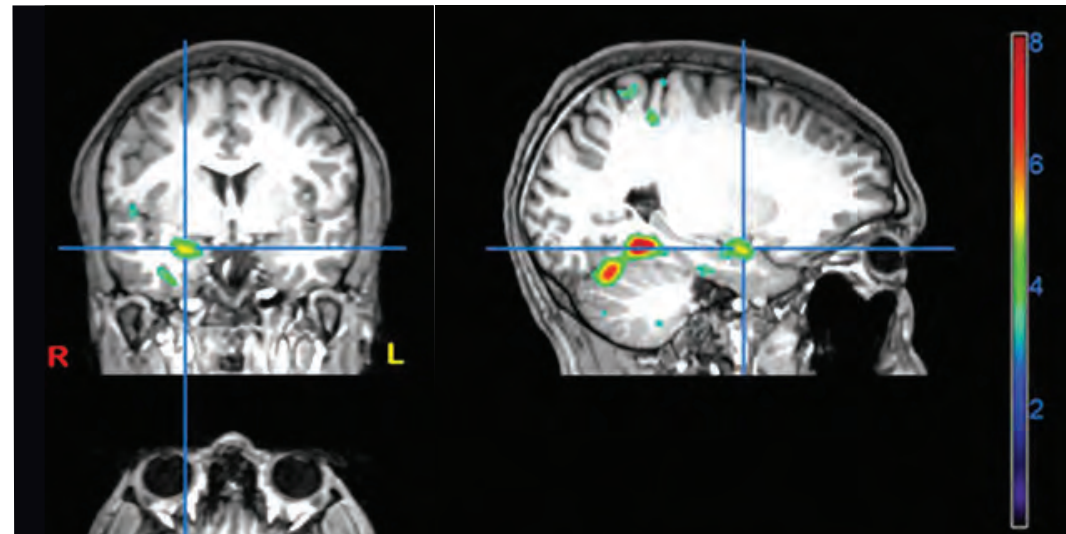

\section{Visual Spatial Memory}

\section{Figure 4}

FMRI activation associated with visuospatial memory performance. The activation occurs on both sides of the brain, primarily in the hippocampus (crosshair) and parahippocampus. The activation is usually stronger in the hemisphere that is not dominant for language (n.b., the right hemisphere), as is the case here.

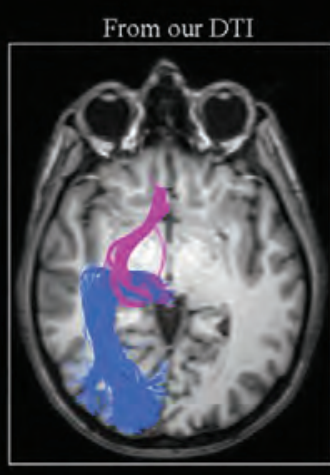

From Dissection Study

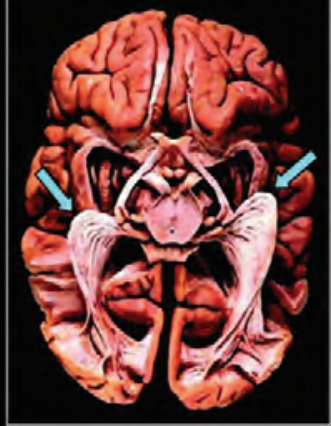

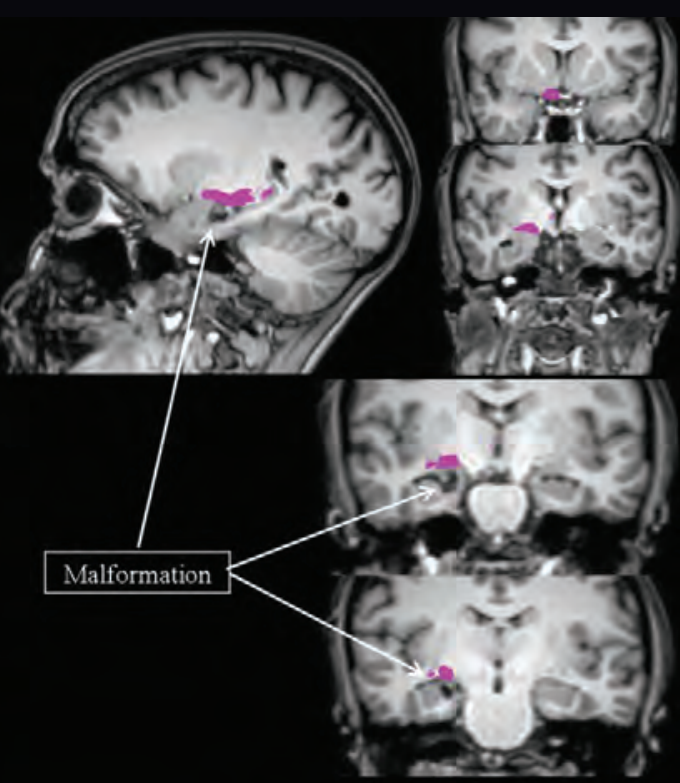

Figure (a)

Optic Tract

Optic Radiation

\section{Figure 5}

Diffusion tractography of the optic tract and optic radiation in a patient with a cortical malformation. 


\section{Clinical Neuroimaging}

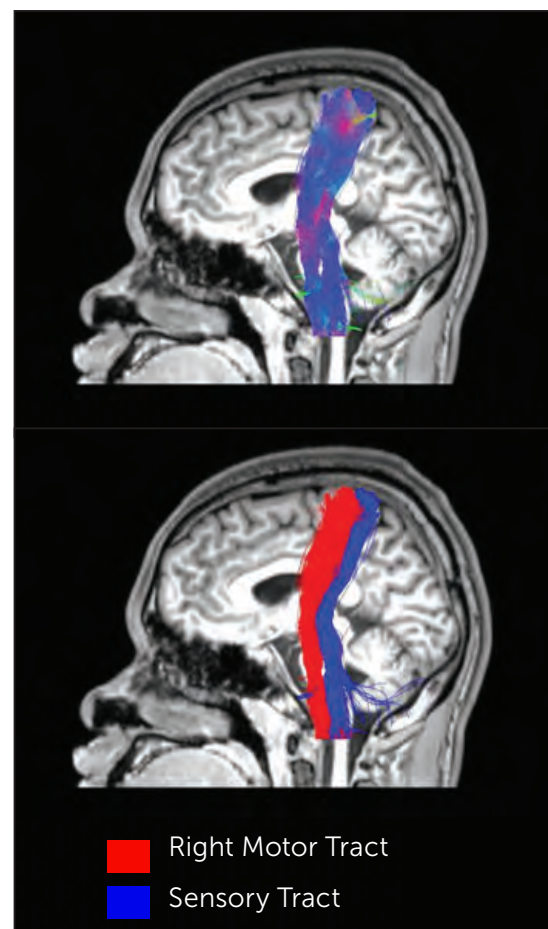

\section{Figure 6}

Diffusion tractography showing the likely location of motor and sensory white matter tracts emanating from primary motor cortex.

\section{Task activation with white matter track}
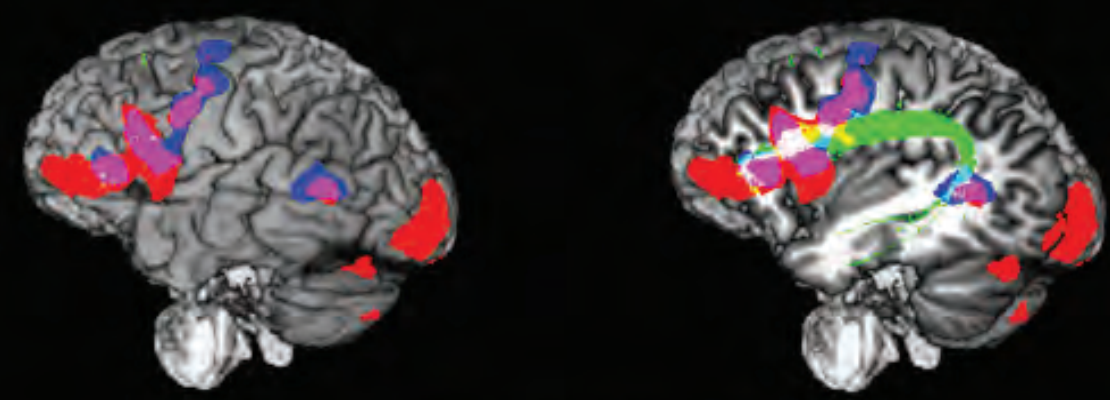

Sentence Completion Task

Verb Generation Task

Left Arcuate Track

\section{Figure 7}

FMRI activation showing the key language areas (Broca's and Wernicke's) in an epilepsy patient. Diffusion tractography was used to depict the likely location of the white matter tract called the arcuate (seen in green) connecting these two language regions.
A

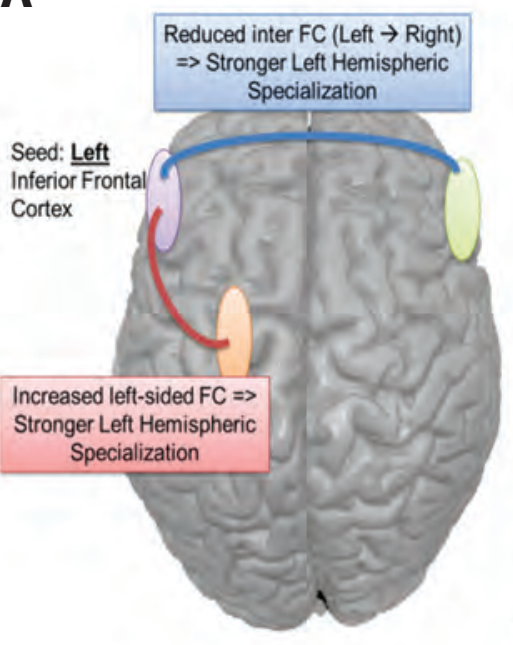

B

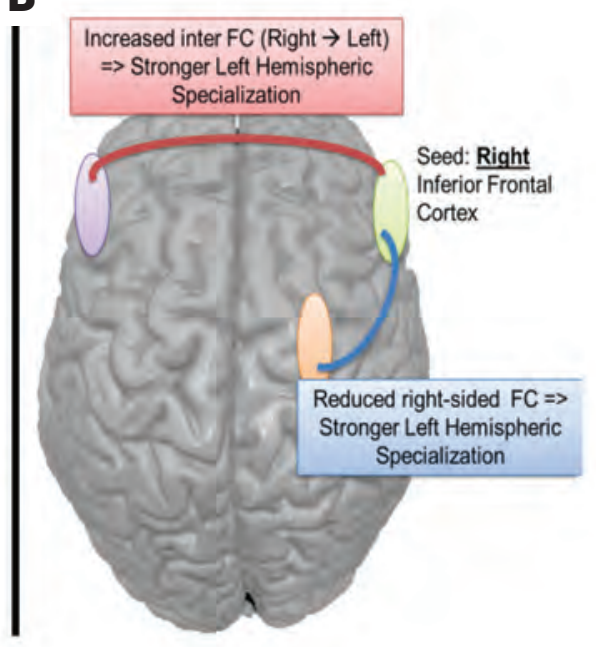

\section{Figure 8}

Resting state functional connectivity patterns associated with stronger left hemisphere specialization for language. The pattern increased (red) and decreased (blue) shown for both seeds involving the left and right inferior frontal cortex applied to both left and right temporal lobe epilepsy patients. Tracy, J., Osipowicz, K., Spechler,P. Sharan, A., Skidmore, C., Doucet, G., and Sperling, M. (2014) Functional Connectivity Evidence of Cortico-Cortico Inhibition in Focal Temporal Lobe Epilepsy. Human Brain Mapping 35(1):353-66. 


\section{Anti-Correlated Activity}

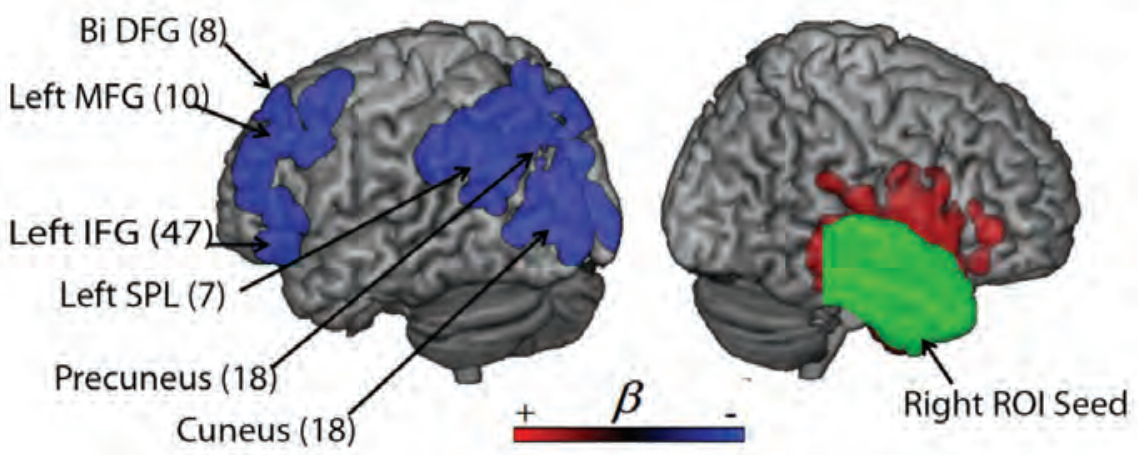

Figure 9

A resting state functional connectivity study showing the areas of anti or negatively correlated activity in the healthy right hemisphere connected to a right temporal lobe seed in a group of patients with seizures emanating from the right temporal lobe. Tracy, J., Osipowicz, K., Spechler, P., Sharan, A., Skidmore, C., Doucet, G., and Sperling, M. (2014) Functional Connectivity Evidence of Cortico-Cortico Inhibition in Focal Temporal Lobe Epilepsy. Human Brain Mapping 35(1):353-66.
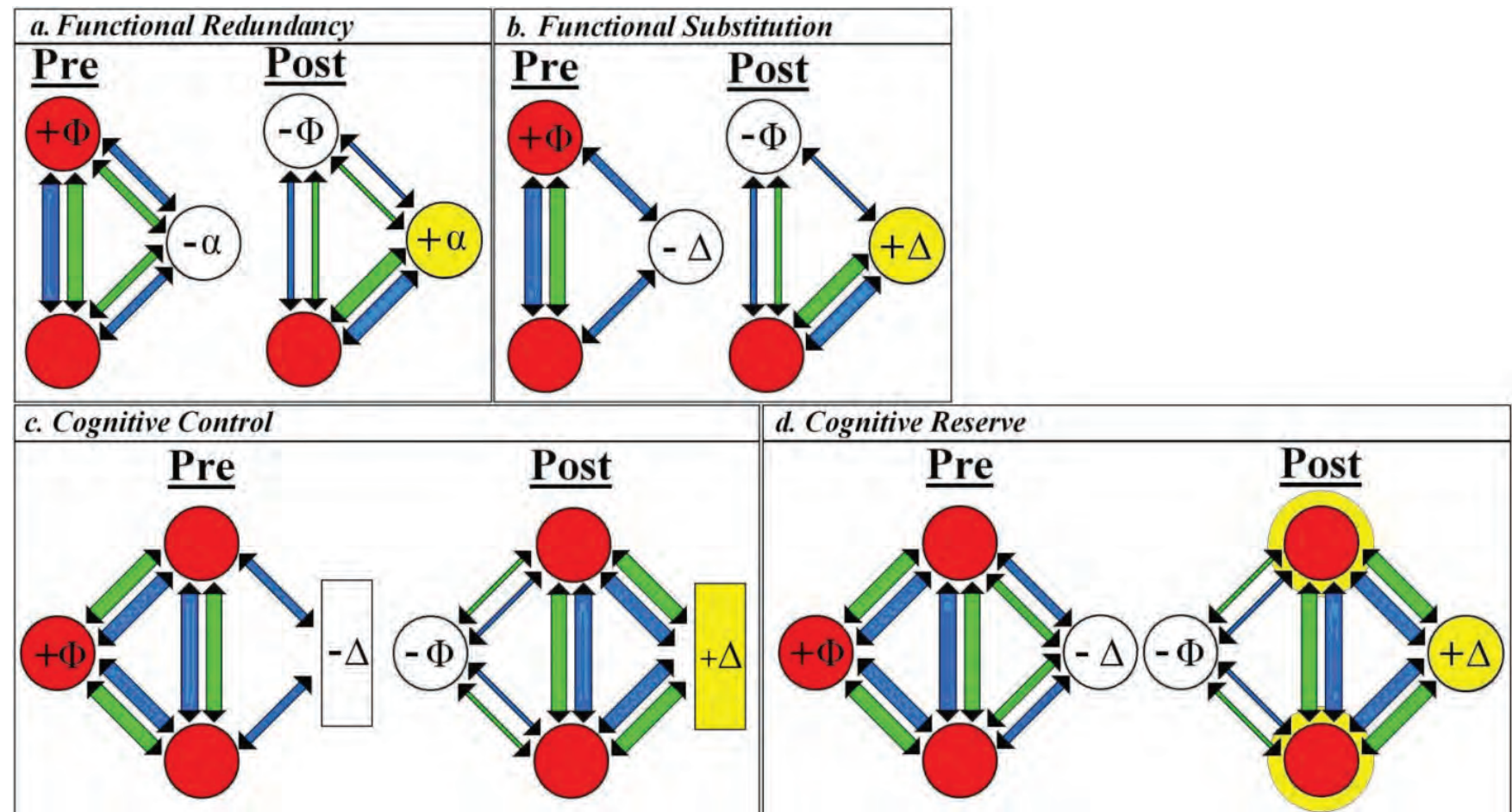

Figure 10

Graphical depiction of multimodal neuroimaging frameworks to identify cognitive reorganization: A. Functional redundancy, B. Functional substitution, C. Cognitive control, D. Cognitive reserve.

Legend: White indicates no fMRI activity in the region. Red circles indicate an area of cognitive functionality as revealed by fMRI. Yellow indicates a new region of fMRI activity, and in the case of Cognitive Reserve also reflects a change in the spatial extent or intensity of activation within the network (n.b., the yellow halo around the red circles of the network). Arrows indicate connectivity, with green lines depicting the rsfMRI findings and blue lines depicting the DTI findings; the arrows indicate the specific brain areas connected. The strength of connectivity is indicated by the thickness of the line with thicker lines indicating stronger connectivity. The + and - indicate the presence or absence of fMRI activity in cases where a change in fMRI occurs across the two time points measured. The Greek letter $\alpha$ indicates a brain area that becomes unmasked at the second scan (e.g., after intervening event such as lesion, brain injury, or surgery); $\Delta$ marks and highlights an area that becomes newly active on the second scan; $\Phi$ indicates a brain area where fMRI activation is lost or drops out of the second scan. Tracy, J. \& Osipowicz, K. (2011) A Conceptual Framework for Interpreting Neuroimaging Studies of Cognitive Recovery. Neurorehabilitation and Neural Repair. 29(4), 331-338 


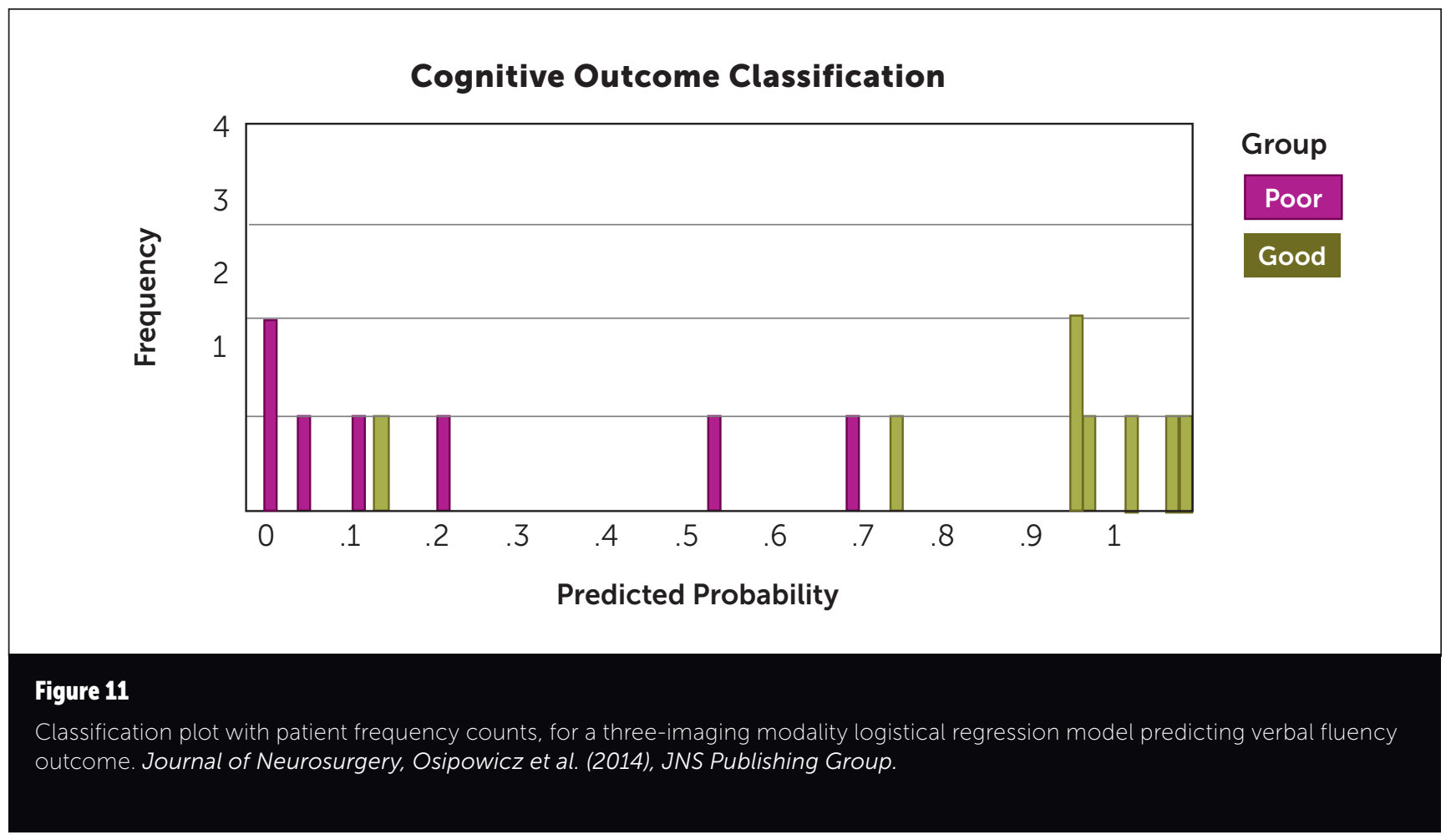

\section{REFERENCES}

1. Doucet, G., Pustina, C., Skidmore, C., Sharan A., Sperling, M., and Tracy, J. (2014) Restingstate functional connectivity predicts the strength of hemispheric lateralization for language processing in temporal lobe epilepsy and normals. Human Brain Mapping. DOI: $10.1002 / \mathrm{hbm} .22628$.

2. Osipowicz, K., Sharan, A., Sperling, M., and Tracy, J. (2015) fMRI, Resting State, and DTI Predict Verbal Fluency Outcome Following Resective Surgery for Temporal Lobe Epilepsy. Journal of Neurosurgery, 25, 1-9.

3. Pustina, D., Avants, B., Sperling, M., Gorniak R., He, X., Doucet, G., Barnett, P., Mintzer, S., Sharan, A., \& Tracy, J. (2015). Predicting the laterality of temporal lobe epilepsy from PET, MRI, and DTI: A multimodal study. Neurolmage: Clinical, 9, 20-31.

4. Tracy, J. \& Doucet, G. (2015). Resting-State Functional Connectivity in Epilepsy: Growing Relevance for Clinical Decision Making. Current Opinion in Neurology. 28(2):158-165
5. Tracy, J. (2015). Commentary on "Network Analysis for a Network Disorder: The emerging role of graph theory in the study of epilepsy, by Bernhardt et al.", Epilepsy \& Behavior, 50, 160-161.

6. Tracy, J., Osipowicz, K., Spechler, P., Sharan, A., Skidmore, C., Doucet, G., and Sperling, M. (2014) Functional Connectivity Evidence of Cortico-Cortico Inhibition in Focal Temporal Lobe Epilepsy. Human Brain Mapping 35(1):353-66

7. Tracy, J. \& Osipowicz, K. (2011) A

Conceptual Framework for Interpreting Neuroimaging Studies of Cognitive Recovery. Neurorehabilitation and Neural Repair. 29(4), 331-338. 